Reprinted from Journal of Applied Physics, Vol. 30, No. 6, 803-807, June, 1959

Copyright 1959 by the American Institute of Physics

Printed in U. S. A.

\title{
Experimental Verification of Theoretical Relations between Total Gas Absorptivities and Total Gas Emissivities for $\mathrm{CO} \dagger$
}

\author{
U. P. OPPENHEIM* \\ Daniel and Florence Guggenheim Jet Propulsion Center, California Institute of Technology, Pasadena, California
}

(Received October 20, 1958)

\begin{abstract}
An experimental study has been carried out for the purpose of verifying theoretical predictions concerning the relations between total absorptivities and total emissivities for transparent gases. The experimental conditions have been designed in such a way as to permit a direct check of theoretical relations derived for (a) nonoverlapping dispersion lines and (b) spectral lines with sufficient pressure broadening to permit neglect of the rotational fine structure. A comparison between the emissivities derived from absorptivity measurements and emissivities calculated from the spectroscopic constants of $\mathrm{CO}$ shows a good agreement (within $20 \%$ for most of the temperature range).

The apparatus for the measurement of total gas absorptivities is described. Results for total absorptivities of carbon monoxide measured at temperatures between 300 and $500^{\circ} \mathrm{K}$ are given and total emissivities have been calculated in the temperature range of 300 to $1600^{\circ} \mathrm{K}$. The range of total gas pressure is 0 to 515 psia.
\end{abstract}

\section{INTRODUCTION}

$T$ HE direct experimental determination of total emissivities for heated gases is notably difficult. ${ }^{1,2}$ For this reason, it is of interest to consider the development of indirect experimental procedures which yield equivalent data with simpler facilities. Recent theoretical studies ${ }^{3,4}$ on the relations between gas absorptivities and emissivities have shown that it is possible to obtain estimates for total gas emissivities on relatively cool gases by using a hot light source, provided the spectral intensity distribution of the vibration-rotation bands is known independently. We have verified the theoretical predictions for two important models on $\mathrm{CO}$, viz., for nonoverlapping dispersion lines and for rotation-vibration bands without rotational fine structure. We believe that judicious use of our procedure, in conjunction with careful theoretical interpretation of results, will permit more accurate emissivity determinations at elevated temperatures than are possible by the use of direct emission measurements.

As part of a larger program on the determination of gas emissivities at various temperatures and pressures, an apparatus has been constructed in which total gas absorptivities can be measured in the 0 to $700 \mathrm{psi}$ pressure range at temperatures between 300 and $1000^{\circ} \mathrm{K}$. Absorptivities were measured with the help of a greybody source the temperature of which could be varied between 300 and $1600^{\circ} \mathrm{K}$.

The total absorptivity of a gas for blackbody radi-

$\dagger$ This work was supported by the United States Office of Naval Research under Contract No. Nonr-220(03), NR 015401.

* On leave from the Weizmann Institute of Science, Rehovoth, Israel.

${ }^{1}$ H. C. Hottel, "Radiant heat transmission," in Heat Transmission, edited by W. H. McAdam (McGraw-Hill Book Company, Inc., New York, 1954), third edition, Chap. IV.

${ }^{2}$ W. Ullrich, Ph.D. thesis, Massachusetts Institute of Technology, Cambridge, 1935.

3 S. S. Penner and A. Thomson, J. Appl. Phys. 28, 614 (1957).

${ }^{4}$ W. J. Hooker and S. S. Penner, J. Appl. Phys., (to be published); D. Olfe and S. S. Penner, J. Appl. Phys. (to be published); Penner, Olfe, and Thomson, ASME Symposium on Thermal Properties, Purdue University, February, 1958 (to be published). ation is defined as the fraction of radiant energy absorbed by the gas. Theoretical expressions ${ }^{3,4}$ relate the total absorptivity of the gas at temperature $T_{g}$ for radiation from a source at temperature $T_{s}$ to the total emissivity of the gas at the source temperature $T_{s}$. It is therefore possible to obtain total emissivities for different gas temperatures from total absorptivities observed at different source temperatures. In the present experiments the gas temperature was from 300 to $500^{\circ} \mathrm{K}$.

Although a number of total absorptivity and emissivity measurements have been reported in the literature, ${ }^{1}$ it was felt that an extension and revision of these measurements with modern instrumentation was needed, particularly if the experimental conditions could be chosen in such a way as to provide a direct experimental check of theoretical predictions. Most of the early emissivity and absorptivity measurements were made on flowing gas samples. The test cell in which the optical observations were made had no windows to confine the gas, which entered at the center of the cell and was drawn off at the ends. This procedure led to uncertainties in the emissivity data. Another difficulty was caused by the fact that the source radiation was not modulated in these experiments, with the result that the absorptivity measurements had to be corrected for radiation emitted from the gas.

In the present study, observations were made on a static column of gas confined in a cell having two periclase $(\mathrm{MgO})$ windows. These windows are transparent in the wavelength range from 0.3 to $7 \mu$ and are extremely hard and refractory. The incident radiation was modulated by a chopper situated between the source and the test cell. In this way, emission of radiation from the gas was eliminated from the detector output.

\section{DESCRIPTION OF THE APPARATUS USED FOR MEASURING TOTAL GAS ABSORPTIVITIES}

The apparatus consisted basically of three parts: a gas handling system, an optical system for radiation 


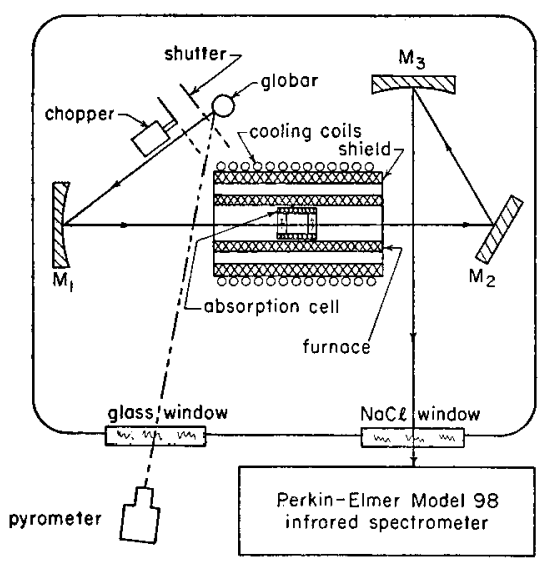

Fig. 1. Schematic diagram of apparatus for measuring total absorptivity. $M_{\mathrm{t}}$, spherical illuminating mirror; $V_{2}$, plane mirror; $\mathrm{M}_{3}$, off-axis parabolic mirror.

measurements, and a temperature controlled test chamber. Both the optical system and the test chamber were enclosed in a vacuum (see Fig. 1). The light source consisted of a water-cooled globar obtained from the Perkin-Elmer Company. Light from the globar was focused by mirror $M_{1}$ on the absorption cell. After traversing the cell the light was reflected from a plane mirror $M_{2}$ and condensed with the help of the parabolic mirror $M_{3}$. A sodium-chloride window in the wall of the vacuum tank enclosing the source and the test cell assembly transmitted the light from the parabolic mirror and permitted it to fall on the entrance slit of a Perkin-Elmer Model 98 monochromator. A thermocouple detector was used in the monochromator. The monochromator was used for inspection of the purity of the gas under study and for detection of residual gases in the path of the radiation. For total absorptivity measurements the radiation was allowed to reach the detector of the monochromator without passing through the prism. The monochromator could be used conveniently for measurements of both spectral and total absorptivity, after it was modified in the following way. A plane mirror was mounted in the parallel beam leaving the parabolic mirror which illuminates the prism of the monochromator. When the additional plane mirror was in a position normal to the beam, no radiation could pass through the prism and the exit slit received the radiation in undispersed form. With this arrangement total radiation measurements were recorded. By swinging the added plane mirror out of the beam, the instrument could be used for spectral absorption measurements.

The radiation was modulated at $13 \mathrm{cps}$ with a standard Perkin-Elmer chopper. The chopper assembly with its rectifying contacts was placed in the vacuum a short distance from the light source. A shutter, which was operated from outside the vacuum chamber, could be raised into the beam in a position between the light source and the chopper.

Commercially available gas supplies were used.
Mixtures of the absorbing gas and an inert gas $\left(\mathrm{He}, \mathrm{N}_{2}\right.$, or A) were prepared in a high-pressure cell where the gases were mixed by using a mechanical stirrer. Pressures up to 1000 psi could be maintained in the mixing cell.

Pressure readings were performed by the use of an Ashcroft test gauge Model 1850 for the pressure range of 0 to $1000 \mathrm{psig}$, with a precision of $\pm 2.5 \mathrm{psig}$. In the pressure range of 0 to 75 psia, a compound Crosley gauge Model AAO was used with a precision of \pm 0.5 psia. A Pirani vacuum gauge (Consolidated Electrodynamics Company Type 2201-0.3) was used for estimating the pressure in the range of 0 to $1 \mathrm{~mm}$ of mercury. The best vacuum was obtained with a Kinney pump Model KC-5 and corresponded to a pressure of $5 \times 10^{-3} \mathrm{~mm}$ of mercury.

The mixing chamber was connected to the absorption cell inside the vacuum tank by a line which passed through the wall of the tank. The absorption cell was machined from pure nickel and had an over-all length of $6 \mathrm{~cm}$. Two periclase windows with a diameter of 25 $\mathrm{mm}$ and a thickness of $6 \mathrm{~mm}$ were situated at opposite ends of the cell. They were kept at a distance of $3 \mathrm{~cm}$ by a nickel spacer. The periclase material for the windows was obtained from Norton Company in the form of large "magnorite" crystals. These were cut and ground to the desired shape by the E.C.L. Laboratories in Pasadena. The windows were sealed against the walls of the test cell with Teflon or Flexitallic gaskets. The free central area of the windows was $13 \mathrm{~mm}$ in diameter.

The absorption cell was situated in a closely fitting electric furnace of cylindrical shape which was $60 \mathrm{~cm}$ long and was wound with 20 -gauge Nichrome wire. The furnace was surrounded by a cylindrical shield of zirconium oxide which was provided with a water-cooled jacket. The temperature inside the furnace was monitored with a pair of Chromel-Alumel thermocouples which were connected to a Leeds and Northrop Speedomax $\mathrm{H}$ temperature control unit. Two additional Chromel-Alumel thermocouples were used to measure the temperature inside the furnace. The temperature in the vicinity of the test cell was constant to within $\pm 5^{\circ} \mathrm{C}$.

\section{OPERATING PROCEDURE}

The tank containing the optical system was evacuated to a pressure of $50 \times 10^{-3} \mathrm{~mm}$ of $\mathrm{Hg}$ and the monochromator was flushed with dry $\mathrm{N}_{2}$. The furnace was heated to the desired temperature and the temperature of the source was set to the desired value. The spectrum of the light source was recorded with the help of the spectrometer and was continuously examined in the regions of atmospheric absorption. The $2.7 \mu$ band of $\mathrm{H}_{2} \mathrm{O}$ and the $4.3 \mu$ band of $\mathrm{CO}_{2}$ were gradually eliminated as the optical path was purged of these two gases. Usually no absorption was present in the globar spectrum after an hour of pumping and flushing. The monochromator was then prepared for taking total radiation measurements by interposing the plane mirror 
in the parallel beam inside the monochromator and by opening the slits to a width of $2 \mathrm{~mm}$. The temperature of the light source was determined and its total emissivity was recorded on the spectrometer. The gasmixing system and the absorption cell were then evacuated to a pressure of $5 \times 10^{-3} \mathrm{~mm}$ of $\mathrm{Hg}$. A gas mixture was prepared at about 700 psig in the mixing chamber and bled into the test cell. The resulting total pressure in the test cell was 500 psig. The decrease in light transmission through the cell accompanying the introduction of the gas into the optical path was recorded on the spectrometer. The pressure in the test cell was decreased to 1 atmos and a new level of transmitted radiation was recorded. In this way the gas mixture was used for making both high-pressure (overlapping-line spectrum) and low-pressure (nonoverlapping-line spectrum) absorptivity measurements. Finally the cell was evacuated and the original source intensity was again obtained. The zero point of the recorder scale was determined by interposing the shutter in the light beam. The accuracy of the recorded intensities was $\pm 0.5 \%$.

A series of such measurements for various gas mixtures at constant source and furnace temperatures was taken. The source temperature was then changed and a new series of gas mixtures was allowed into the test cell. In this way a large range of operating conditions was covered. Finally, the furnace temperature was changed and the previously described measurements were repeated.

\section{PERFORMANCE OF THE APPARATUS}

In discussing the performance of the apparatus a number of points deserve special attention.

\section{Stability of the Light Source}

The globar required a well-regulated current in order to produce a stable radiation output. When the globar voltage was provided through a Variac which received its input from a Sola constant voltage transformer, the radiation output varied in an irregular manner. Good stability was obtained when a number of 10-25 Amperite ballast tubes was put in series with the globar. The ballast tubes kept the current constant over a wide range of input voltages. A variable shunt across the globar was used to change the source temperature; the total current was kept constant while the globar current was set at any desired lower level.

\section{Blackness of the Light Source}

Recent studies ${ }^{5}$ have established that, within close limits, the globar is a graybody with an emissivity of 0.75 in the 0.6 to $15 \mu$ wavelength region. To a first approximation, the emissivity is independent of the temperature at which the globar is operated. The total

\footnotetext{
${ }^{5}$ (a) J. E. Stewart and J. C. Richmond, J. Research Natl. Bur. Standards 59, 405 (1957). (b) W. Brügel, Z. Physik 127, 400 (1950).
}

absorptivity $\alpha$ of a gas at temperature $T_{g}$, irradiated by a blackbody source at temperature $T_{s}$, is given by the relation,

$$
\alpha\left(T_{s}, X, T_{g}\right)=\frac{1}{\sigma T_{s}{ }^{4}} \int_{0}^{\infty} R^{0}\left(\omega, T_{s}\right)\left[1-\exp -\left(P_{\omega, T_{g}} X\right)\right] d \omega,
$$

where $\sigma$ is the Stefan-Boltzmann constant, $R^{0}\left(\omega, T_{s}\right)$ is the spectral blackbody radiancy at the temperature $T_{s}$ and in the wave-number interval between $\omega$ and $\omega+d \omega$, $X$ is the optical depth of the gas, and $P_{\omega, T_{g}}$ represents its absorption coefficient at the wavenumber $\omega$ and the temperature $T_{g}$. It is obvious that the expression for $\alpha$ is the same for a gray source as for a blackbody.

Since the emissivity of the globar is known, its temperature $T_{s}$ may be determined with the help of an optical pyrometer. The brightness temperature $T_{b}$ obtained with such a pyrometer is related to the true temperature $T_{s}$ by the relation

$$
\frac{1}{T_{s}}=\frac{1}{T_{b}}+\frac{\lambda}{c_{2}} \ln \epsilon_{\lambda}
$$

where $\epsilon_{\lambda}$ is the emissivity of the light source at the wavelength $\lambda$, and $c_{2}$ is the second radiation constant. In this expression $T_{\mathrm{s}}$ is not a very sensitive function of $\epsilon_{\lambda}$. The pyrometer, which employs a filter of wavelength $\lambda=0.65 \mu$, therefore gives a fairly accurate estimate of $T_{s}\left( \pm 3^{\circ} \mathrm{C}\right.$ in the range of 700 to $\left.1600^{\circ} \mathrm{C}\right)$ even if the emissivity is not known with a precision greater than \pm 0.05 .

The measurements of $T_{b}$ were carried out with a Leeds and Northrop optical pyrometer Model 8622-C, which operated in the temperature range between 760 and $2800^{\circ} \mathrm{C}$. Below $760^{\circ} \mathrm{C}$, the temperature $T_{s}$ was determined with a Chromel-Alumel thermocouple attached to the globar.

\section{Long Wavelength Cutoff}

The wavelength selective properties of the optical system will now be examined. It is apparent from the definition of $\alpha$ that true values of the total absorptivity will be recorded by the detector only if the following conditions are met: a. The light source is black (or grey). b. The optical system between source and detector is neutral, i.e., it has a transmissivity which is independent of wavelength. c. The detector is black (or gray).

The first and last conditions were met in the present apparatus. However, the periclase windows are opaque beyond $7 \mu$. The fraction of blackbody radiation which lies beyond $7 \mu$ is easily calculated from Planck's radiation formula. ${ }^{6}$ The apparent absorptivity data may then be corrected by taking this effect into account. The maximum correction to $\alpha$ amounts to $40 \%$.

\footnotetext{
${ }^{6}$ N. A. Lowan and G. Blanck, J. Opt. Soc. Am. 30, 70 (1940).
} 


\section{Influence of Slit Width}

When the monochromator was used for recording total radiation, the amount of light falling on the thermocouple detector was many orders of magnitude larger than the corresponding amount of dispersed radiation. It was necessary to close down the slits of the monochromator to widths of less than $50 \mu$ in order to avoid overloading of the thermocouple. However, for these small slit widths the absorptivity was found to depend on the value of the slit width. The smaller the slit width, the smaller the value of the recorded absorptivity. It is reasonable to assume that strong diffraction of long wavelengths takes place at the stops of the system, with the result that a smaller fraction of long wavelength radiation was received by the detector than was contained in the original beam. The apparent dependence of absorptivity on slit width disappeared when very wide slits $(2 \mathrm{~mm})$ were used and the radiation was attenuated by aperture stops on the optical elements of the monochromator.

\section{Emission of Radiation by the Chopper}

If the chopper is heated to a temperature $T_{c h}$ during operation, its modulated radiation will reach the detector and cause a negative signal. Assuming the chopper to radiate as a blackbody, the net modulated flux falling on the detector is proportional to

$$
F_{1}=\sigma T_{s}^{4}-\sigma T_{c h}{ }^{4}
$$

when it is receiving radiation of a blackbody source at temperature $T_{s}$. With the shutter (at temperature $T_{s h}$ ) between the source and the chopper a zero reading is recorded which is proportional to

$$
F_{0}=\sigma T_{s h}^{4}-\sigma T_{c h}{ }^{4} .
$$

Here the shutter is also assumed to have blackbody properties. The difference

$$
F_{1}-F_{0}=\sigma T_{s}^{4}-\sigma T_{s h^{4}}
$$

is usually taken as a measure of the intensity of the light source. When the shutter is removed and a gas of total absorptivity $\alpha\left(T_{s}, X, T_{\theta}\right)$ is placed in the path of the radiation, the detector receives a flux proportional to $(1-\alpha) \sigma T_{s}^{4}-\left(1-\alpha^{\prime}\right) \sigma T_{s h}{ }^{4}$, where $\alpha^{\prime}$ indicates the total gas absorptivity $\alpha^{\prime}\left(T_{s h}, X, T_{q}\right)$ for a source of temperature $T_{s h}$. The measured fractional transmission is now given by

$$
\frac{(1-\alpha) \sigma T_{s}^{4}-\left(1-\alpha^{\prime}\right) \sigma T_{s h}{ }^{4}}{\sigma T_{s}^{4}-\sigma T_{s h}{ }^{4}} .
$$

It is seen that this expression reduces to the desired value of $(1-\alpha)$ only if $T_{s h} \ll T_{s}$. A careful experimental evaluation has shown that the emission of radiation from both the chopper and the shutter was negligibly small compared with $\sigma T_{*}^{4}$ for the temperatures employed in our studies.

\section{TOTAL ABSORPTIVITY MEASUREMENTS FOR CO}

The gas used in the present study was C.P. grade $\mathrm{CO}$ obtained from the Matheson Company. It had a nominal purity of $96.8 \%$ and contained $0.36 \%$ of $\mathrm{CO}_{2}$, $1.0 \%$ of $\mathrm{N}_{2}, 0.97 \%$ of $\mathrm{H}_{2}$, and $0.8 \%$ of saturated hydrocarbons. The small amount of $\mathrm{CO}_{2}$ gave rise to a strong absorption band at $4.3 \mu$. Since no absorption bands other than the bands under study are permissible in a total absorptivity determination, the $\mathrm{CO}_{2}$ had to be completely removed. This was achieved by forcing the gas through a tube containing ascarite, which proved to remove $\mathrm{CO}_{2}$ very effectively even when a large volume of gas was passed over it in a very short time interval.

A set of total absorptivity measurements on $\mathrm{CO}-\mathrm{A}$ mixtures was made at a total pressure of 1 atmos. Previous studies have established ${ }^{7}$ that no appreciable

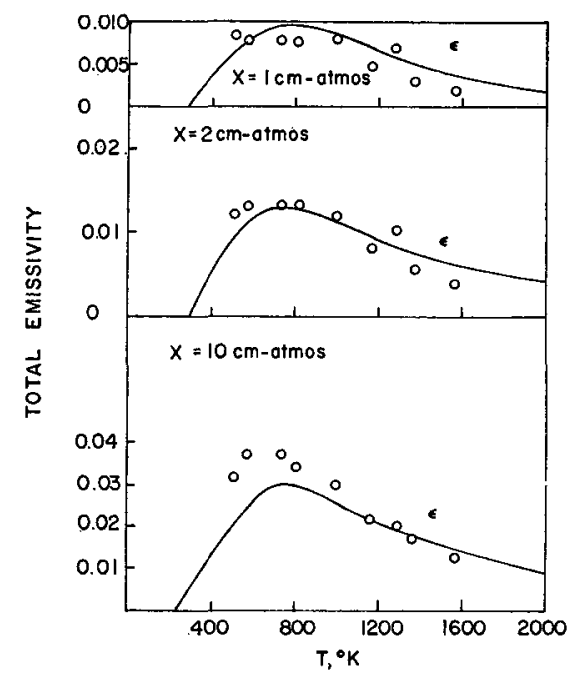

FIG. 2. Total emissivities of $\mathrm{CO}$ as a function of temperature at a total pressure of 1 atmos; emissivities based on theory for nonoverlapping lines ${ }^{8}: \epsilon$; emissivities computed from observed absorptivities: 0 .

overlapping occurs between lines of the vibrationrotation bands at this pressure for optical thicknesses between 0 and $10 \mathrm{~cm}$-atmos. The total absorptivity was measured as a function of $T_{s}, T_{g}$, and $X$. From these data the total emissivity was calculated using the following relation for nonoverlapping dispersion lines with regular spacing and constant collision half-width ${ }^{4}$ :

$$
\alpha\left(T_{s}, X, T_{o}\right)=\epsilon\left[T_{s}, X \frac{T_{s}}{T_{g}}\right] .
$$

The results of the calculation are presented in Fig. 2. The total emissivity is plotted as a function of absolute temperature for optical thicknesses of 1,2 , and $10 \mathrm{~cm}$ atmos. The circles indicate theoretically calculated $\mathrm{CO}$ emissivities based on a nonoverlapping line model with

\footnotetext{
${ }^{7}$ S. S. Penner and D. Weber, J. Chem. Phys. 19, 1351 (1951).
} 
a half-width of $0.031 \mathrm{~cm}^{-1}$-atmos ${ }^{-1} \cdot{ }^{8,9}$ It is interesting to note that, apart from some points in the diagram for $X=10 \mathrm{~cm}$-atmos, the experimental results agree with the theoretical curve within the limits of experimental accuracy. The experimental data of Ullrich ${ }^{2}$ have not been included since they were higher by about a factor of two.

A second set of observations on CO-A and $\mathrm{CO}-\mathrm{He}$ mixtures was made at total pressures of 35 and 18 atmos, respectively. It has been established ${ }^{7}$ that for gas pressures above 18 atmos and at room temperature the rotational fine structure of the vibration-rotation bands may be neglected if the optical thickness is above $1 \mathrm{~cm}$-atmos. Total emissivity was calculated by using the following relation between total absorptivity and total emissivity for the case of overlapping rotational lines [reference 3, Eq. (24)]:

$$
\alpha\left(T_{s}, X, T_{g}\right)=\left(\frac{T_{g}}{T_{s}}\right)^{\frac{3}{2}} \epsilon\left[T_{s},\left(\frac{T_{s}}{T_{g}}\right)^{\frac{3}{2}} X\right] .
$$

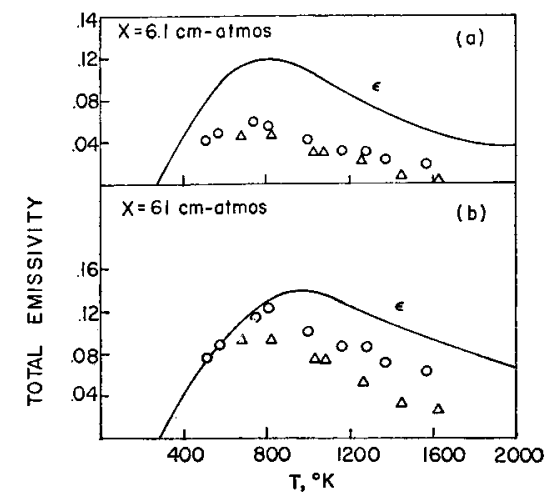

Fig. 3. Total emissivities of $\mathrm{CO}$ as a function of temperature; emissivities based on theory for overlapping rotational lines ${ }^{10}: \epsilon$; emissivities computed from observed absorptivities of $\mathrm{CO}-\mathrm{A}$ mixtures at a total pressure of 35 atmos: 0 ; emissivities computed from observed absorptivities of $\mathrm{CO}-\mathrm{He}$ mixtures at a total pressure of 18 atmos: $\Delta$.

The results of this calculation are presented in Figs. 3 and 4 . The solid curves represent theoretically cal-

${ }^{8}$ S. S. Penner, J. Appl. Phys. 23, 825 (1952).

$\checkmark$ D. Weber and S. S. Penner, J. Chem. Phys. 21, 1503 (1953).

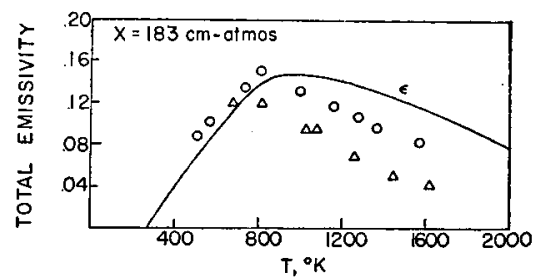

Frg. 4. Total emissivities of $\mathrm{CO}$ as a function of temperature; emissivities based on theory for overlapping rotational lines ${ }^{10}: \epsilon$; emissivities computed from observed absorptivities of $\mathrm{CO}-\mathrm{A}$ mixtures at a total pressure of 35 atmos: 0 ; emissivities computed from observed absorptivities of CO-He mixtures at a total pressure of 18 atmos: $\Delta$.

culated total emissivities for $\mathrm{CO}$, assuming overlapping lines..$^{10}$ Also indicated are the present results for argonbroadened $\mathrm{CO}$ at a total pressure of 35 atmos and the results for helium-broadened $\mathrm{CO}$ at a total pressure of 18 atmos. Reference to Figs. 3 and 4 shows that reasonably good agreement is observed for high optical depths although above $900^{\circ} \mathrm{K}$ the experimental points fall below the theoretical curves. This is to be expected since the assumption of overlapping lines fails to apply at high temperatures for which the number density of emitters is low and the half-width of the (collisionbroadened) lines is small compared with the half-width at room temperature. The assumption of overlapping lines fails to apply also at low optical depths, causing the experimental points to fall below the theoretical curve in Fig. 3(a). It is interesting to note that the emissivities obtained using helium as a pressurizing gas lie below those using argon, in agreement with the relative half-width estimates for these two broadening agents. ${ }^{9}$ This effect is less noticeable below $900^{\circ} \mathrm{K}$ since in this temperature range and for the total pressures used, both gases are adequately effective in smearing out the fine structure of the spectrum.

\section{ACKNOWLEDGMENTS}

The present study was suggested by Professor S. S. Penner, to whom I am indebted for many valuable discussions. The apparatus described in this report evolved from an earlier version constructed by Mr. D. Weber at the Jet Propulsion Laboratory in 1954. 